\title{
Using Fast Frequency Hopping Technique to Improve Reliability of Underwater Communication System
}

\author{
Jan H. SchmidtiD \\ Faculty of Electronics, Telecommunications and Informatics, Gdańsk University of Technology, \\ ul. Narutowicza 11/12, 80-233 Gdańsk, Poland; jan.schmidt@pg.edu.pl
}

Received: 30 December 2019; Accepted: 7 February 2020; Published: 10 February 2020

\begin{abstract}
Acoustic underwater communication systems designed to work reliably in shallow coastal waters must overcome major limitations such as multipath propagation and the Doppler effect. These restrictions are the reason for the complexity of receivers being built, whose task is to decode a symbol on the basis of the received signal. Additional complications are caused by the low propagation speed of the acoustic wave in the water and the relatively narrow bandwidth. Despite the continuous development of communication systems using coherent modulations, they are still not as reliable as is desirable for reliable data transmission applications. This article presents an acoustic underwater communication system that uses one of the varieties of the spread spectrum technique i.e., the fast frequency hopping technique (FFH). This technique takes advantage of binary frequency-shift keying (BFSK) with an incoherent detection method to ensure the implementation of a system whose main priority is reliable data transmission and secondary priority is the transmission rate. The compromised choice of parameters consisted of the selection between the narrow band of the hydroacoustic transducer and the maximum number of carrier frequency hops, which results from the need to take into account the effects of the Doppler effect. In turn, the number of hops and the symbol duration were selected adequately for the occurrence of multipath propagations of an acoustic wave. In addition, this article describes experimental communication tests carried out using a laboratory model of the FFH-BFSK data transmission system in the shallow water environment of Lake Wdzydze/Poland. The test results obtained for three channels of different lengths are discussed.
\end{abstract}

Keywords: shallow coastal waters; reliable underwater acoustic communication; fast frequency hopping technique; bit error rate

\section{Introduction}

In the water environment, due to the fact that electromagnetic waves are strongly suppressed, acoustic waves are used to implement the underwater communication systems. Systems whose most important task is reliable operation in shallow coastal waters must overcome numerous limitations. One of the most important factors is multipath propagation. Its occurrence is the result of signal reflections from the water surface and the bottom (characteristic of horizontal channels) and any obstacles present in water reservoir. The acoustic wave may also be refracted and dispersed over the inhomogeneities of the medium. As a result, inter-symbol interference (ISI) is observed, which is undesirable in communication systems. Another important factor is the Doppler effect caused by the movement of the transmitter and/or receiver relative to each other. Although these adverse factors also occur in other communication systems, which are implemented using a different transmission medium, it is in the water environment and using acoustic waves where they become particularly emphasized. This is due to the low speed of propagation of the acoustic waves and the narrow available bandwidth, which directly results in limiting the throughput of the hydroacoustic channel [1]. Their seasonal 
variability is also observed, which is an additional difficulty in clearly defining the acoustic parameters of the underwater communication channels [2,3].

The already developed and commercially used hydroacoustic modems usually use a carrier frequency below $100 \mathrm{kHz}$, while for technological reasons, hydroacoustic transducers offer a bandwidth on the order of several kilohertz. All of these restrictions taken together are the reason for the complexity of the built receivers, whose task is to decode a symbol on the basis of the received signal. It is generally known that the underwater acoustic channel is one of the most demanding channels for the implementation of modern digital communication. This applies in particular to transmission through a horizontal channel occurring in shallow coastal waters.

In wireless underwater communication, various modulation techniques are used, mainly frequency and phase modulation of the carrier wave [4-8]. They are used in both single-carrier and multi-carrier systems. The use of non-coherent modulations allows the undesirable effects of multipath propagation and the Doppler effect to be effectively overcome, especially in a horizontal underwater channel, where they provide transmission rates from several dozen to several hundred bits per second (bps) $[9,10]$. These modulations are most often used in devices requiring long-term operation from battery power sources, due to the simple design of the receiver. Coherent modulations are used for systems operating in the environment of deep waters as well as vertical channels. Coherent modulations use the available frequency band more efficiently, offering higher transmission rates, but require a more complex receiver that performs adaptive correction of the transmission channel characteristics [11,12]. Compared to non-coherent modulations, they require a signal with a higher signal-to-noise ratio for proper operation. In general, coherent modulation techniques remain less reliable than is desirable. Obtaining improving transmission reliability or higher transmission rates for this modulation method is possible as a result of transmission over many independent propagation paths, which is obtained by using multiple hydroacoustic antennas [13]. However, for compact applications, this means inconvenient hardware system complications. Another of the modulation techniques, the spread spectrum technique, is widely used in military ground communication systems due to its ability to work with a low signal-to-noise ratio and its good tolerance to multipath propagation and system jamming. In addition, the use of channel coding together with interleaving reduces the bit error rate.

This article is a development of research carried out by the author over the past few years on reliable underwater communication systems for work in shallow coastal waters [14,15]. Its main subject is to specify the parameters for the FFH technique and to present the results of experimental tests on the lake. The compromised choice of parameters consisted of the selection between the narrow band of the hydroacoustic transducer and the maximum number of carrier frequency hops, which results from the need to take into account the effects of the Doppler effect. In turn, the number of hops and the symbol duration were selected adequately for the occurrence of multipath propagations of an acoustic wave.

\section{Features of a Reliable Underwater Communication System}

Spread spectrum techniques were originally developed for applications in military systems due to their low probability of intercept and high resistance to jamming signals, i.e., they make it difficult for the opponent to eavesdrop on transmissions and unauthorized sources transmitting false information-interfering with communication through interference with the receiver system. In addition, it is possible to share the frequency band with many types of conventional modulations with or without minimal interference.

This technique is based on the statement: The more the noise dominates the signal, the wider the band the signal must occupy to receive it correctly. It results from Shannon's theorem on channel bandwidth with a specified bandwidth $B$ and signal-to-noise ratio (SNR) [16]. This technique significantly expands the bandwidth used, while allowing you to work with a much smaller ratio of signal strength to noise power, which is done by converting the narrowband information signal to a signal with a much wider band $[17,18]$. 
There are many varieties of spread spectrum technique, among which the most typical are [19]:

- Direct Sequence Spread Spectrum technique (DSSS) [20],

- Frequency Hopping Spread Spectrum technique (FHSS),

- Time Hopping Spread Spectrum technique (THSS),

- $\quad$ Chirp Spread Spectrum technique (CSS) [21].

One of these varieties is considered in the article, i.e., FHSS, as a technique that will ensure reliable operation of an underwater communication system. The next part of the chapter is devoted to discussing the principle of the system with this technique, and then the implementation of the receiver and the assumptions of this technique in the case of counteracting the limitation of the communication channel.

Communication systems utilizing a frequency hopping technique most often use frequency-shift keying (FSK) with an incoherent detection method [19]. A diagram of a typical system with the details of the modulator and demodulator is shown in Figure 1.

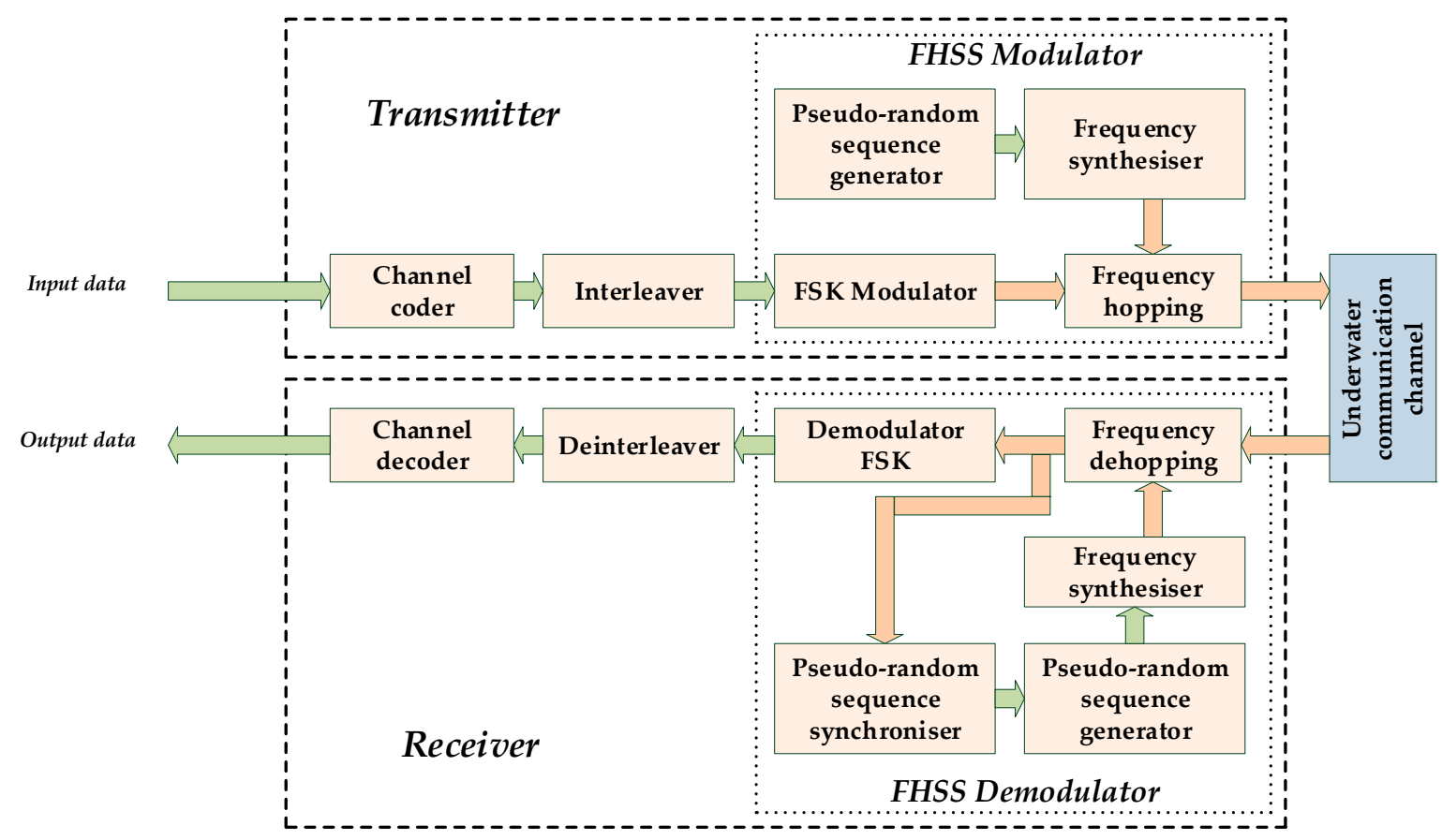

Figure 1. Scheme of data transmission system with frequency hopping technique with details of the modulator and demodulator.

In the FHSS technique, all available channel bandwidth are divided into adjacent frequency subchannels. The carrier frequency between the subchannels is switched using a selected code sequence, which is known in both the transmitter and the receiver. The L-length code sequence controls the frequency synthesizer in each symbol interval $T_{S}$. In the transmitter, the sent symbol is subjected to channel coding and interleaving, and then goes to the FSK modulator, which sends the symbol with duration $T_{S}$, and gives the signal the appropriate frequency for the subchannel depending on the FSK modulation order used. The signal thus generated is then placed in the appropriate frequency subchannel by means of a frequency conversion system for a time equal to the time of single hop time $T_{H}$ (or dwell time). Then, after amplification, such a signal goes through the hydroacoustic transducer to the underwater channel. On the receiving side, the amplified signal in the preamplifier goes to the receiver. In the receiver, the signal is reduced to the baseband in which FSK demodulation occurs. The synchronization signal necessary to maintain the synchronization of the code sequence generator is obtained from the received signal by the code sequence synchronizer system [19]. 
When the carrier frequency changes every few bits of data stream, it is a system with slow frequency hopping technique (SFH).

\subsection{Fast Frequency Hopping Technique}

When $T_{S} / T_{H}>1$, i.e., the change of carrier frequency occurs repeatedly during the duration of the $T_{S}$ data symbol, then it is a system with the fast frequency hopping technique (FFH). Figure 2 shows a graphic operation diagram of the fast frequency hopping technique for an example of a code sequence pattern.

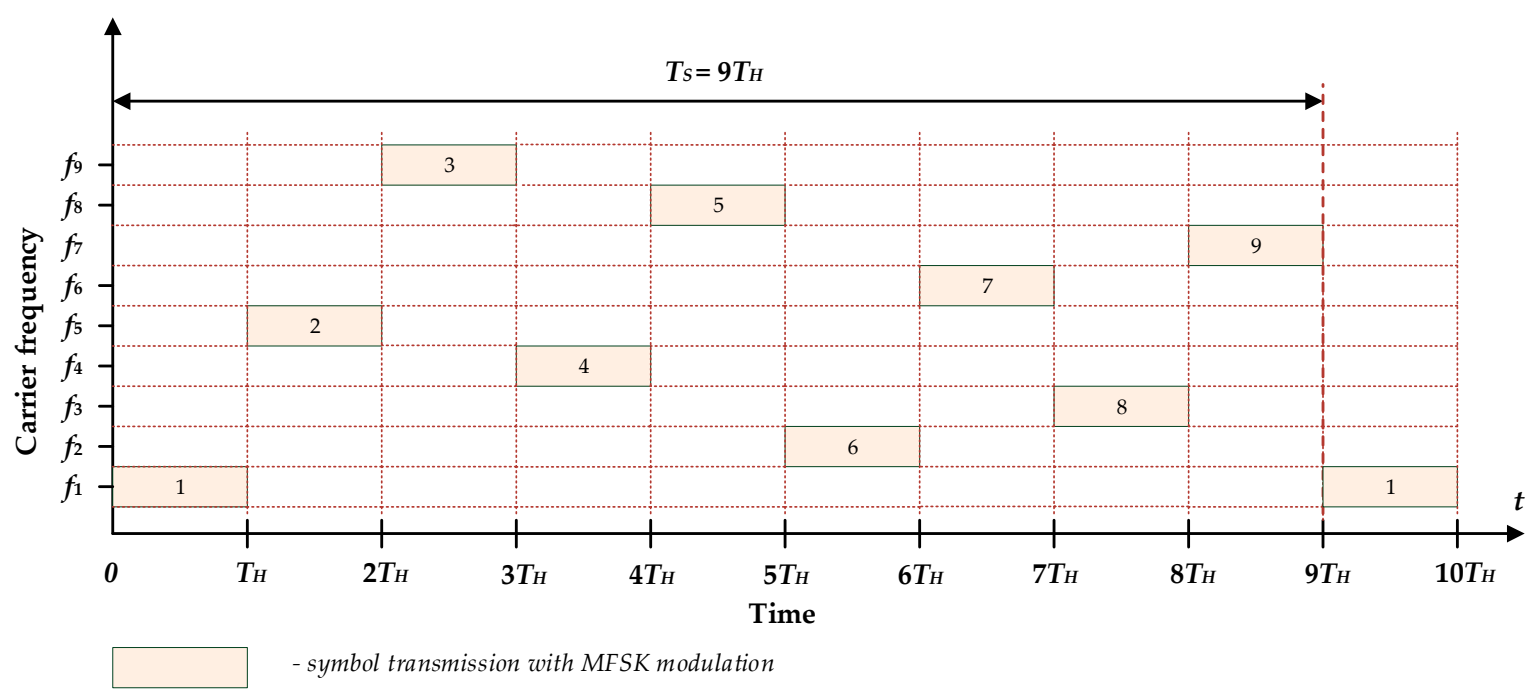

Figure 2. Operation diagram of the fast frequency hopping technique for an example code sequence pattern with a length of $9\left(T_{S}=9 \cdot T_{H}\right)$.

As mentioned, non-coherent FSK frequency modulation is usually used in this method. The use of coherent modulations would be difficult due to the phase coherence at the time in which the carrier frequency is changed according to the pattern determined by the code sequence.

In the FFH technique, the hop frequency is equal to $f_{H}=1 / T_{H}$. In the case of non-coherent detection, to ensure the required orthogonality between the tones of the FSK modulation signal, a minimum distance between them being a multiple of $f_{H}$ should be used. This property ensures that no crosstalk occurs with the remaining hop frequency bands.

An important parameter characteristic of the spread spectrum techniques, the $N_{S S}$ spreading factor, for the FFH technique corresponds to the length of the code sequence $L$ and is equal to $T_{S} / T_{H}$.

The form of the transmitted low-pass FFH-FSK signal can be described by the Formula (1) [22],

$$
s_{n}(t)=\sqrt{\frac{2 E_{S}}{T_{S}}} \cos \left[2 \pi\left(f_{l}+f_{m}\right) t+\varphi_{n}\right]
$$

where:

$$
\left[(n-1)+\frac{l-1}{L}\right] T_{S} \leq t \leq\left[(n-1)+\frac{l}{L}\right] T_{S}, \quad 1 \leq n \leq N, \quad 1 \leq l \leq L, \quad 0 \leq m \leq M-1,
$$

while $E_{S} / T_{S}$ stands for the power of the transmitted symbol signal, $T_{S}$ is the duration of the symbol signal, $l$ is the hop number, $f_{l}$ is the intermediate frequency for the selected band of the $l$-th frequency hop, $f_{m}$ is the $m$-th significant frequency, $\varphi_{n}$ is an unknown random phase associated with the transmitted symbol, $n$ is the number of the transmitted symbol, $M$ is the modulation order of FSK, and $N$ is the number of all symbols to send. 
The necessary system bandwidth of a transmission system using the FFH-FSK technique is determined by the Equation (3):

$$
B_{F F H-F S K}=\frac{L(M+1)}{T_{H}}[\mathrm{~Hz}]
$$

The FFH system performs the transmission and reception of symbols based on diversity techniques [23]. In general, diversity techniques contributes to ensure redundancy. Some of the received symbols can be treated as more reliable than others, and based on them, the right decision about the received symbol can be made. The receipt of each of the redundant symbols must be independent in order for the diversity technique used to meet its objectives.

The diversity technique used in the FFH technique is a hybrid of time and frequency diversity, which can be seen in Figure 2. Typically, time diversity accomplishes repeated transmitting and receiving of the same symbol over time. However, the independent reception of the symbol in the faded channel requires that each symbol transmission with $T_{S}$ duration be time separated by a time greater than the coherence time $T_{c}$ of the channel. In turn, frequency diversity typically performs the transmission and reception of the same symbol on multiple carrier frequencies at the same time, but adjacent carrier frequencies must be separated by a band larger than the coherence bandwidth $B_{c}$ of the channel. However, the time diversity and that implemented in the FFH technique operate sequentially, because the redundant symbols are received individually at a specific time and use the available carrier frequencies. For a fixed bandwidth per signal, the order of diversity $L$ increases with the amount of redundancy. Similarly, the average signal energy $(\cdot L)$ also increases, but the transmission rate $(/ L)$ decreases.

With regard to the stream of transmitted symbols, the diversification used in the FFH technique can be interpreted in a simplified way as the use of repetition coding [24,25]. Using hard-decision decoding in the receiver, a decision is made about which symbol was received for each hop with duration $T_{H}$. If we assume that the example probability of receiving an erroneous symbol is $P_{S}=0.3$ for a single $T_{H}$ hopping time, which is equivalent to a single symbol transmission $(L=1)$, then the probability of receiving an erroneous symbol for the odd values of symbol repetition (=values of the order of diversity) can be determined using Formula (4) [25]. The probability is presented in Figure 3 for several dozen consecutive values of $L$ repetitions and it follows that as the repetitions increase, the $P_{b}$ value decreases. At this stage of considerations, regarding the quality of data transmission, it is reasonable to suppose that the FFH technique will provide the desired $P_{b}$ value with the optimal number of $L$ replicates.

$$
P_{S}(L)=\sum_{i=\frac{L+1}{2}}^{L}\left(\begin{array}{c}
L \\
i
\end{array}\right) P_{S}{ }^{i}\left(1-P_{S}\right)^{L-i}
$$

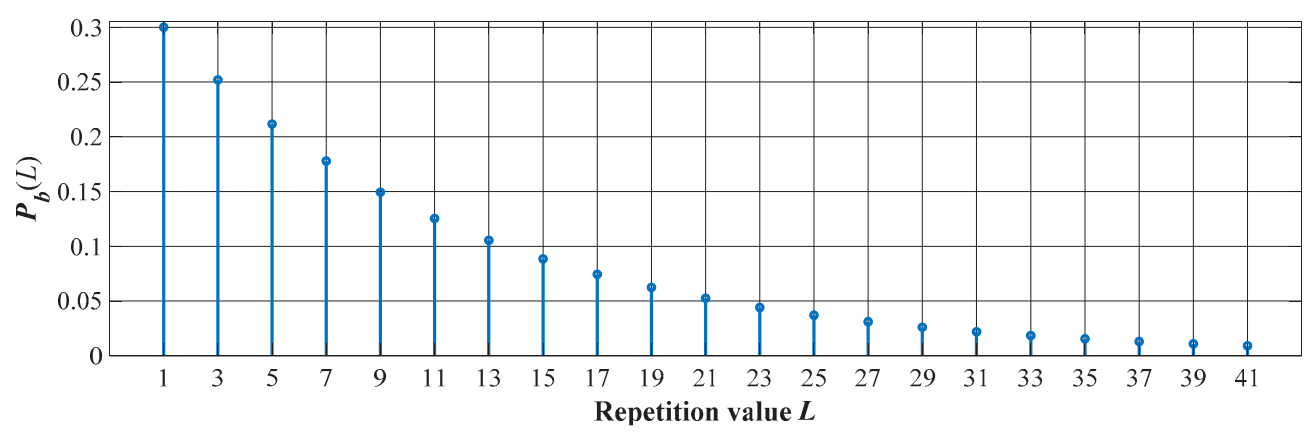

Figure 3. The value of the probability error of the received symbol for different repetition values (=order of diversity). 


\subsection{Implemented Receiver}

The structure of the FFH-FSK receiver implemented in the considered data transmission system is shown in Figure 4. Initially, the received signal $r(t)$ is subjected to analogue-to-digital conversion, and then the selected band signal is reduced to the baseband of the specified FFH-FSK width. The next operations lead to a discrete signal spectrum, which is used to determine the power density spectrum of the discrete signal for subsequent $T_{H}$ durations. The obtained spectrum covers the entire FFH-FSK band, i.e., it covers all possible branches corresponding to the significant frequencies of FSK modulation for each of the $L$ possible hops, hence the number of all branches analyzed is $L \cdot M$. Next, the receiver extracts the values of the spectral lines $Y_{m, k}$ and determines the decision values $Z_{m}$ according to the selected reception diversity algorithm $(m=0, \ldots, M-1 ; k=1, \ldots, L)$, in pursuance of the assumptions of soft-decision decoding. The basic condition for the proper functioning of the FFH technique receiver is its synchronization with the frame of the transmitted symbol, and then the algorithm is zeroed and all decision variables $Z_{m}$ are worked out.

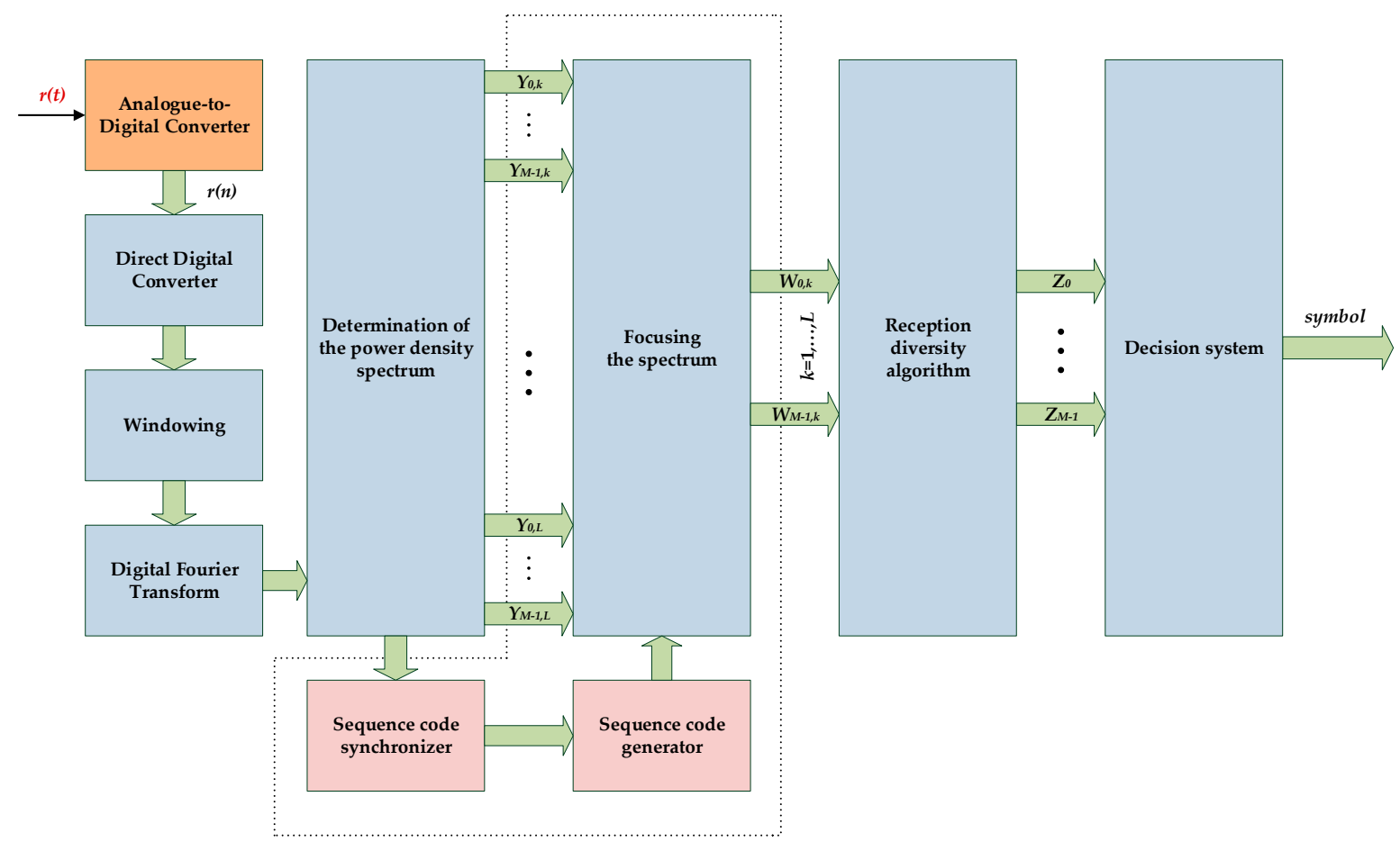

Figure 4. Structure of the implemented FFH-FSK receiver.

In the first stage, for the next $L$ hop times $T_{H}$ that occur for transmissions of a single symbol, the values of the $Y_{m, k}$ bands are extracted for each of the $M$ FSK branches and $L$ of possible hops, creating a matrix with dimensions $(M+L) \cdot L$. In the second stage, based on the obtained matrix, the operation of focusing the spread spectrum symbol signal is performed by selecting the value of the spectral lines from $M$-th FSK branches for the next $L$ hop times $T_{H}$. Transmissions of a single symbol are $L$ hops, and the next hop $k$ is in accordance with the hop pattern determined based on the fixed code sequence. As a result of these actions, a matrix of values $W_{m, k}$ with dimensions $M \cdot L$ is created, which is used by the reception diversity algorithm to determine the decision value of $Z_{m}$, independently for each branch of $m$. The obtained decision values of $Z_{m}$ allow the final decision to be made, i.e., to assign the appropriate FSK symbol to the obtained decision values $Z_{m}$. 
The square-law combining algorithm was used as the reception diversity algorithm in the FFH technique, which sums $L$ values of $W_{m, k}$ from each MFSK branch, according to Formula (5). It assumes that independent transmission channels have the same gain [23].

$$
Z_{m}=\sum_{k=1}^{L} W_{m, k}{ }^{2}
$$

\subsection{Counteracting the Effects of multipath Propagation and the Doppler Effect}

To meet the condition of implementing frequency diversity, the occurrence of independent fading in the frequency-selective channel should be assumed, when the frequency spacing between the two signals is greater than the coherence bandwidth $B_{c}$ of the channel $\left(\Delta f_{F F H-F S K}=1 / T_{H}>B_{c}\right)$ [3]. Therefore, the planning of frequency hops in conjunction with the design of the appropriate hop pattern resulting from the generated code sequence is important for the FFH system under consideration. However, thoughtful design of the hop pattern must take into account that the length of the code sequence, and thus the number of possible hops, is limited by the specificity of the hydroacoustic channel and the useful bandwidth of available transducers, typically several $\mathrm{kHz}$. This fact also significantly limits the design of a more complex pattern design.

In general, in order to assume the frequency non-selective channel (also known as flat fading channel), it is required to use spacing between neighbouring hop frequencies smaller than the coherence bandwidth $B_{c}$ of the channel $\left(\triangle f_{F F H-F S K}<B_{c}\right)$. In contrast, the relatively broad bandwidth of the FFH system can be modelled as a group of independent narrowband frequency non-selective channels using a stochastic process with slow Rician or Rayleigh fading [23]. The term slow fading means that the fading is constant during a single hop and then changes in signal amplitudes caused by the presence of fading are invariable for a single hop. For the number $L$ of frequency hopping per transmission and reception of a single symbol, the effective duration of the symbol is defined as $T_{S}=(L-1) \cdot T_{H}$, and hence in the time domain, the above assumption about the frequency non-selectivity channel can be written as $T_{s}=(L-1) \cdot T_{H}>T_{m}$.

For $T_{H}>T_{m}$ the channel has flat fading and, therefore, allows the impact of multipath effects to be neglected, while for $T_{H}<T_{m}$ the channel is frequency-selective and the FFH technique aims to remove the effect of multipath effects on the direct path signal. Because the FFH system receiver after a reception time equal to the hop time $T_{H}$ jumps to the new hop frequency when the multipath components begin to reach the receiver, the arriving multipath components occupy a different band from the currently received signal, so the effect of unwanted interference should be insignificant. During reception equal to the hop time $T_{H}$, at least one non-fading signal associated with the direct path is processed.

The condition for meeting the requirements for the implementation of time diversity in the case of a channel with fast fading is to ensure that the time interval $T_{c c}$ between the transmission of subsequent symbol signals with the duration of $T_{H}$ at the same hop frequency is longer than the coherence time $T_{\mathcal{C}}$ of the channel. This means mutual independence in the time domain for two hops at the same frequency. At the same time, the time interval $T_{c c}$ can be interpreted as the time at which each of the hopping frequencies used is unused, which allows the multipath components present in the hopping band to be suppressed. This assumption applies provided that the maximum delay spread $T_{m}$ is shorter than the discussed period $T_{c c}$.

To ensure the occurrence of slow fading, which is more convenient as opposed to fast fading, the hop time $T_{H}$ must be shorter than the coherence time $T_{c}$ of the channel $\left(T_{H}<T_{c}\right)$, thus the condition $\Delta f_{\text {FFH-FSK }}>B_{d}$ must be fulfilled by analogy.

To prevent the influence of the Doppler effect, which occurs as a Doppler deviation, the bandwidth reserved for a particular signal should be determined with a specified significant frequency. Therefore, the condition $B_{d}<<B_{s}$ must be met, where $B_{d}$ is the maximum Doppler spread and $B_{s}$ is the band of the transmitted symbol. 


\section{Results of Underwater Experimental Tests}

In order to determine the quality of data transmission, experimental tests were conducted on 4-5 May 2017, on Lake Wdzydze/Poland near the headquarters of the Hydroacoustic Research Station of the Sonar Systems Department/Gdańsk University of Technology. Figure 5 shows the general configuration of the measuring stations.

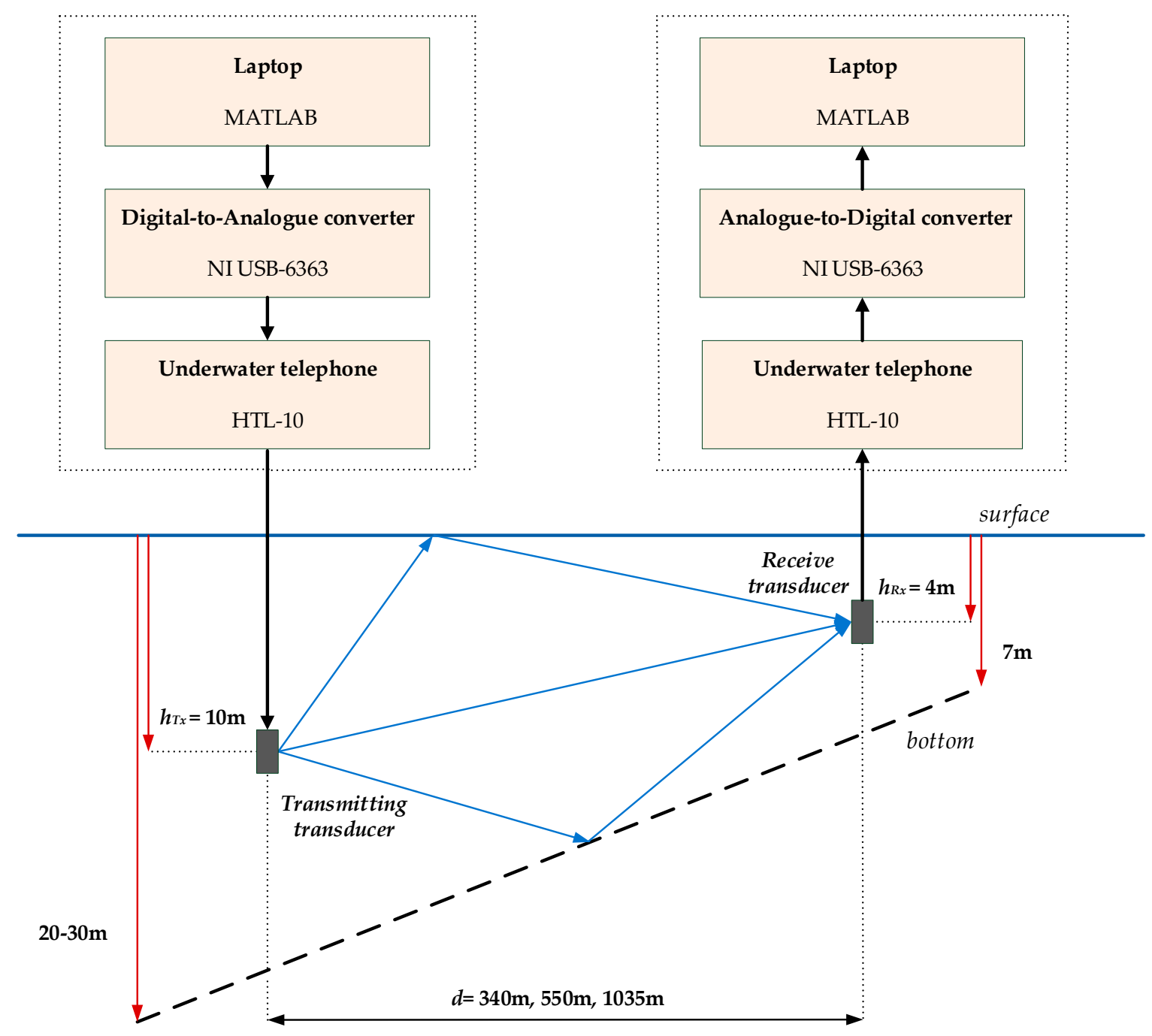

Figure 5. Configuration of measuring stations.

Each of the transmitted signals was generated in the MATLAB environment, and the conversion of this signal to analogue form was performed using the digital-to-analogue converter available in the National Instruments USB-6363 recording and generating device. The signal was then amplified and transmitted through an underwater channel using a HTL-10 underwater telephone [15]. Its design is based on the assumptions of programmable radio (SDR-Software-Defined Radio). This assumption makes it possible to use it for research purposes with various signals in the bands from $1 \mathrm{kHz}$ to $60 \mathrm{kHz}$. The HTL-10, which I am a co-author of the hardware and software part, has been successfully used by the Polish Navy on its helicopters for several years. It uses hydroacoustic antenna of the OKA-2M dipping sonar that is designed to search for submarines because the helicopter devices allow it to descend into the water using a several-hundred-meter-long cable when the helicopter is hovering.

The transmitting measuring station was placed on board a motor boat. The same measuring equipment was used on the receiving side, but it was configured differently. The receiving measuring 
station was placed in a measuring container placed on a floating platform moored to a bridge with a fixed position during each of the measuring sessions. The received signal was sampled and archived. The transmitting transducer was lowered from the motor boat to a depth of $h_{T x}=10 \mathrm{~m}$ regardless of the depth of the water reservoir, which for all transmitting positions of the measuring stations was from 20 to $30 \mathrm{~m}$. The receiving transducer was lowered to a depth of $h_{R x}=4 \mathrm{~m}$, in a place where the water depth was equal to $7 \mathrm{~m}$. In subsequent sessions, the distance $d$ between the transmitting and receiving stations changed and amounted to 340,550 and $1035 \mathrm{~m}$, respectively.

On the receiving side, the analogue signal was subjected to initial analogue processing by the analogue processing unit of the underwater telephone HTL-10. The obtained signal is digitized by an analogue-to-digital converter with a sampling frequency of $f_{s}=200 \mathrm{kHz}$. Then, the digital receiver uses the digital down converter technique (DDC) to directly convert the real passband signal around the selected carrier frequency into a complex baseband signal around zero frequency [26]. In the conversion process, the receiver uses optimized algorithms for digital signal processing.

Before performing the series of measurements, the sound velocity distribution profile was measured using a sound velocity distribution meter. The measured profile and the determination of the acoustic wave propagation paths based on it made it possible to estimate the expected range obtainable of the communication system, which is presented in Figure 6. Propagation paths for the hydroacoustic transducer submerged to a depth of $10 \mathrm{~m}$ were determined. On the presented sound velocity distribution profile in Figure 6a, the sound velocity gradient is negative, hence the drawing of propagation path clearly indicates that sound rays are refracted towards the bottom.

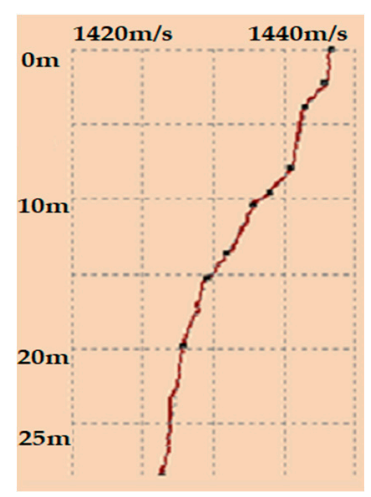

(a)

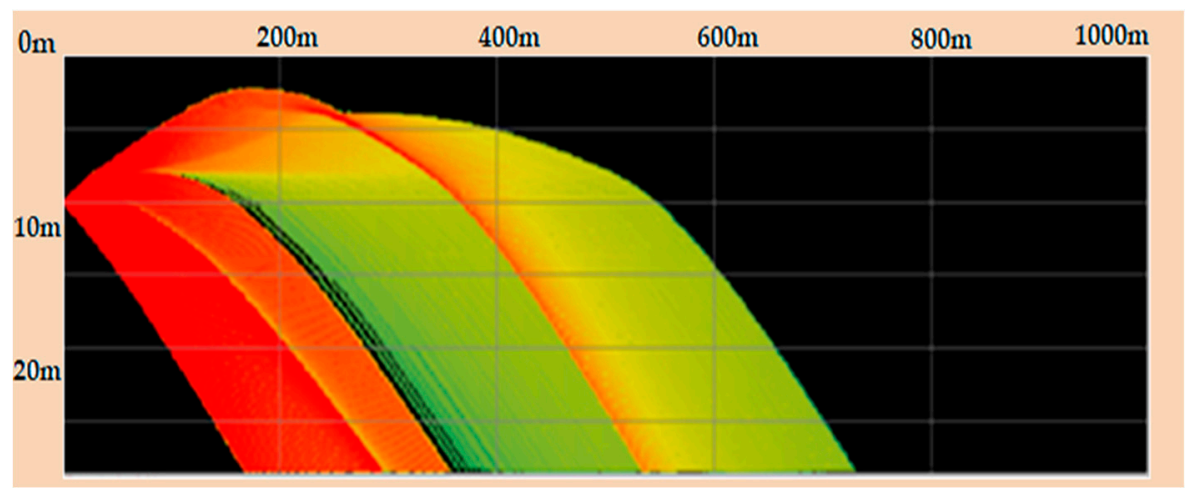

(b)

Figure 6. (a) Measured sound velocity distribution profile; (b) Estimated system range.

In the considered system, the fast frequency hopping technique assumes the use of BFSK modulation due to the availability of a narrow system frequency band of approximately $5000 \mathrm{~Hz}$. In this technique, hops are made between available subbands during the transmission of a single symbol. The jumps are made according to the assumed pattern, and their maximum number in the tests was set at $L=9$. The transmissions of subsequent symbols with the hop duration $T_{H}=\{4 \mathrm{~ms}, 16 \mathrm{~ms}, 64 \mathrm{~ms}\}$ were subjected to experimental tests. The recorded transmissions of individual symbols were used to determine the bit error rate of reception. A sampled signal with a time equal to the duration of a single hop is subjected to analysis based on a periodogram. Tests of the FFH technique with BFSK modulation were carried out for all channel types, i.e., $d=340 \mathrm{~m}, d=550 \mathrm{~m}$ and $d=1035 \mathrm{~m}$ using the following carrier frequency hopping pattern $\left(\left[f_{1} f_{2} f_{3} f_{4} f_{5} f_{6} f_{7} f_{8} f_{9} f_{1} f_{2} f_{3} f_{4} \ldots\right]\right)$. For the $d=550 \mathrm{~m}$ channel, data transmission was performed using pseudo-random hops $\left(\left[f_{1} f_{5} f_{9} f_{4} f_{8} f_{2} f_{6} f_{3} f_{7} f_{1} f_{5}\right.\right.$ $\left.f_{9} f_{4} \ldots\right]$ ]). Experimental studies were carried out under slightly different meteorological conditions, i.e., for $d=340 \mathrm{~m}$ and $d=1035 \mathrm{~m}$, the sea state was 1 , while for $d=550 \mathrm{~m}$ the sea state was 3 . The sea state was determined according to the Douglas sea scale, where the sea state indicates the state of the sea surface depending on the height of the wave. For sea state 1 , wave height is $0.0-0.1 \mathrm{~m}$ and this 
state is referred to as calm-rippled. In turn, for sea state 3, wave height is $0.5-1.5 \mathrm{~m}$ and it is defined as slight. During measurements, the HTL-10 was used, which has limited transmission power adjustment capabilities, which made it impossible to perform a comparative analysis of the received bit error rate values for different SNR values of the received signal. For BFSK modulation, a distance between significant frequencies of $\Delta \mathrm{f}=250 \mathrm{~Hz}$ was determined, which allows the maximum Doppler deviation of $125 \mathrm{~Hz}$ to be taken into account. The distance used requires the use of a subband with a width of $2 \cdot \Delta \mathrm{f}=500 \mathrm{~Hz}$. The system bandwidth, determined on the basis of Formula (3), for a maximum hop count of $L=9$ is $4500 \mathrm{~Hz}$. The measurements were carried out using the carrier frequency $f_{c}=30 \mathrm{kHz}$.

As a result of the analysis of the recorded data transmission signals with the FFH-BFSK technique, in the $d=340 \mathrm{~m}$ channel no transmission errors were found for the considered order of diversity $L=\{3,5,7,9\}$. Of all of the tested channels, this channel was characterized by the shortest maximum delay spread $T_{m}$ of $\sim 2$ ms. Tables 1 and 2, which contain the results of FFH technique tests for $d=550 \mathrm{~m}$ and $d=1035 \mathrm{~m}$ channels, show that only the use of $T_{H}=4 \mathrm{~ms}$ hop time was associated with transmission errors. Errors were observed for the first channel case when the diversity order was set at $L=3$ and $L=5$ and for the second channel at the $L=3$ diversity order. The increase in the order of diversity, as well as the increase in the hop time, caused the transmission to also be error-free in the $d=550 \mathrm{~m}$ channel, the most difficult of the tested channels. The maximum delay spread $T_{m}$ for the first channel was $\sim 7 \mathrm{~ms}$, and for the second one $\sim 9 \mathrm{~ms}$. The differences were probably in the reinforcements of individual multipath components. The above tests were performed for the FFH technique with a standard with successive hops. For longer hop times $T_{H}=\{16 \mathrm{~ms}, 64 \mathrm{~ms}\}$, no errors occurred.

Table 1. Experimental tests results for the $d=550 \mathrm{~m}$ channel (subsequent jumps, $T_{H}=4 \mathrm{~ms}, E_{H} / N_{0}=31 \mathrm{~dB}$ ).

\begin{tabular}{ccc}
\hline Order of Diversity $L$ & Transmission Rate [bps] & $\begin{array}{c}\text { Bit Error Rate } \\
\text { BER }\end{array}$ \\
\hline 3 & 83.3 & 0.024 \\
5 & 50 & 0.019 \\
7 & 35.7 & $<0.001$ \\
9 & 27.7 & $<0.001$ \\
\hline
\end{tabular}

Table 2. Experimental tests results for the $d=1035 \mathrm{~m}$ channel (subseq. jumps, $T_{H}=4 \mathrm{~ms}, E_{H} / N_{0}=31 \mathrm{~dB}$ ).

\begin{tabular}{ccc}
\hline Order of Diversity $\boldsymbol{L}$ & Transmission Rate [bps] & $\begin{array}{c}\text { Bit Error Rate } \\
\text { BER }\end{array}$ \\
\hline 3 & 83.3 & 0.022 \\
5 & 50 & $<0.001$ \\
7 & 35.7 & $<0.001$ \\
9 & 27.7 & $<0.001$ \\
\hline
\end{tabular}

Using the pattern with pseudo-random jumps in the FFH technique for the $d=550 \mathrm{~m}$ channel, the results were obtained. As for the successive hopping case, transmissions with errors were obtained only for the duration of the hop $T_{H}=4 \mathrm{~ms}$ and only for the order of diversification $L=3$.

The use of the pattern with successive hops and the pattern with pseudo-random hops gave similar results of the quality of data transmission expressed as BER, which could be expected due to the small number of possible hops.

Figure 7 presents examples of decision values corresponding to a logical ' 0 ' and a logical ' 1 ' obtained for subsequent hops falling on the transmission of a single symbol ' 0 ' in the case of transmission through the $d=340 \mathrm{~m}$ and $d=550 \mathrm{~m}$ channel. The figure confirms the fact that the channels were characterized by different multipath propagations of acoustic wave. 

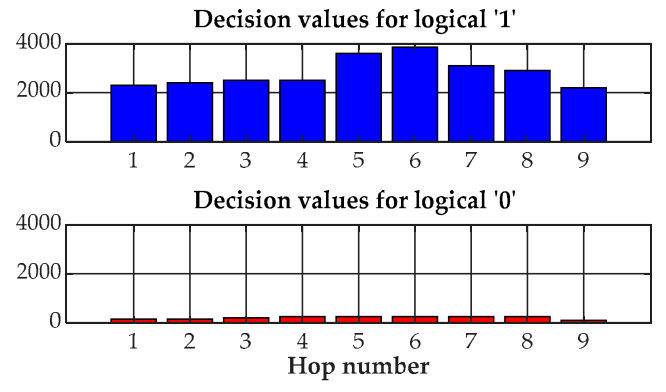

(a)
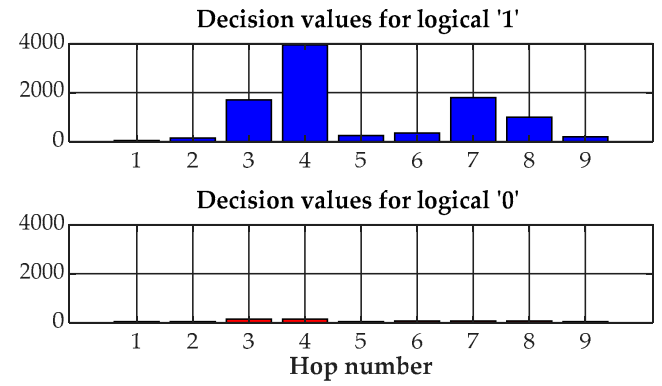

(b)

Figure 7. Examples of decision values corresponding to logical ‘ 0 ' and logical ' 1 ' obtained for successive hops per transmission of a single symbol ' 0 ' for different channels: (a) for $d=340 \mathrm{~m}$ channel; (b) for $d=550$ m channel.

Summarizing the obtained results of the experimental tests carried out on a lake, the FFH technique confirms high efficiency when working in shallow coastal waters. The commercial modems designed to work in horizontal channels that use other modulation techniques allow obtaining similar transmission rates of several dozen to hundreds of bits per second and require the use of strong channel coding. Tests gave an answer on the nature of the errors occurring. During the analysis of the FFH-BFSK technique with $L=\{2, \ldots, 9\}$, no burst errors were observed, only random errors were observed.

\section{Conclusions}

This article is devoted to an underwater acoustic communication system, which has been set to have specific requirements resulting from working in the difficult propagation conditions that occur in shallow coastal waters. The specificity of the work environment of the underwater communication system results in numerous limitations of digital data transmission. The transmitted signal undergoes strong multipath propagation and the influence of the Doppler effect. These phenomena and the narrow frequency band of the system, resulting from the low operating frequency (from several to several dozen $\mathrm{kHz}$ ) and design restrictions of ultrasonic transducers, result in relatively low data transmission rates being achieved. In turn, transmission losses and noise are the main factors that limit the range of the system and determine the choice of operating frequency. An additional significant limitation is the low speed of acoustic wave propagation, which results in a strong delay in the reception of transmitted signals, which in two-way communication limits the effective transmission rate.

An underwater communication system using the fast frequency hopping technique has been described in detail. The influence of acoustic wave propagation conditions in shallow coastal waters on the quality of data transmission in the considered communication system was examined. The results of experimental studies confirmed the thesis that increasing the order of diversity $L$ allowed an improvement of detection conditions to be achieved and thus a reduction in the transmission bit error rate. It can also be stated that the FFH technique is effective in a channel with fading despite the fact that the available operating bandwidth is relatively narrow for a communication system. Unfortunately, an increase in the order of diversity $L$ reduces the system's transmission rate.

Funding: This research received no external funding.

Acknowledgments: Special thanks to Iwona Kochańska and Krzysztof Liedtke for technical support in experimental tests.

Conflicts of Interest: The author declares no conflict of interest in this work.

\section{References}

1. Urick, R.J. Principles of Underwater Sound for Engineers; McGraw: New York, NY, USA, 1983. 
2. Grelowska, G. Study of Seasonal Acoustic Properties of Sea Water in Selected Waters of the Southern Baltic. Polish Maritime Research 2016, 23, 25-30. [CrossRef]

3. Kochanska, I.; Schmidt, J.; Rudnicki, M. Underwater Acoustic Communications in Time-Varying Dispersive Channels. In Proceedings of the 2016 Federated Conference on Computer Science and Information Systems (FedCSIS), Gdańsk, Poland, 11-14 September 2016; pp. 467-474.

4. Kilfoyle, D.B.; Baggeroer, A. The state of the art in underwater acoustic telemetry. IEEE J. Ocean. Eng. 2000, 25, 4-27. [CrossRef]

5. Chitre, M.; Shahabudeen, S.; Freitag, L.; Stojanovic, M. Recent advances in underwater acoustic communications \& networking. In Proceedings of the OCEANS 2008 IEEE, Quebec City, QC, Canada, 15-18 September 2008.

6. Baggeroer, A.B. An overview of acoustic communications from 2000-2012. In Proceedings of the Workshop on Underwater Communications (UComms): Channel Modelling \& Validation, Sestri Levante, Italy, 12-19 September 2012.

7. Zhou, S.; Wang, Z. OFDM for Underwater Acoustic Communications; Wiley: Hoboken, NJ, USA, 2014.

8. Dol, H.S.; Casari, P.; van der Zwan, T.; Otnes, R. Software-defined underwater acoustic modems: Historical review and the NILUS approach. IEEE J. Ocean. Eng. 2017, 42, 722-737. [CrossRef]

9. Garrood, D. Applications of the MFSK Acoustic Communications System. In Proceedings of the OCEANS 81, Boston, MA, USA, 16-18 September 1981. [CrossRef]

10. Schmidt, J.; Zachariasz, K.; Salamon, R. Underwater communication system for shallow water using modified MFSK modulation. Hydroacoustics 2005, 8, 179-184.

11. Stojanovic, M.; Catipovic, J.A.; Proakis, J.G. Phase-coherent digital communications for underwater acoustic channels. IEEE J. Ocean. Eng. 1994, 19, 100-111. [CrossRef]

12. Schmidt, J.H.; Schmidt, A.M.; Kochanska, I. Performance of Coherent Modulation Scheme Used in Acoustic Underwater Communication System. Vib. Phys. Syst. 2019, 30, 1-8.

13. Schmidt, J.H.; Schmidt, A.M.; Kochanska, I. Multiple-Input Multiple-Output Technique for Underwater Acoustic Communication System. In Proceedings of the 2018 Joint Conference Acoustics, Ustka, Poland, 11-14 September 2018. [CrossRef]

14. Schmidt, J.H. Reliable underwater communication system for shallow coastal waters. Hydroacoustics 2014, 17, 171-178.

15. Schmidt, J.H. The development of an underwater telephone for digital communication purposes. Hydroacoustics 2016, 19, 341-352.

16. Shannon, C.E. A Mathematical Theory of Communication. Bell Syst. Techn. J. 1948, 27, 379-423. [CrossRef]

17. Scholtz, R.A. The origins of spread-spectrum communications. IEEE Trans. Commun. 1982, 5, 822-854. [CrossRef]

18. Pickholtz, R.L.; Schilling, D.L.; Milstein, L.B. Theory of spread-spectrum communications-A tutorial. IEEE Trans. Commun. 1982, 5, 855-884. [CrossRef]

19. Haykin, S. Communication Systems, 4th ed.; Wiley: Hoboken, NJ, USA, 2001.

20. Kochanska, I.; Schmidt, J. Simulation of Direct-Sequence Spread Spectrum Data Transmission System for Reliable Underwater Acoustic Communications. Vib. Phys. Syst. 2019, 30, 1-8.

21. Marszal, J.; Rudnicki, M.; Jedel, A.; Salamon, R.; Kochanska, I. Mutual Clutter Suppression Techniques for FM Sonars. Arch. Acoust. 2016, 41, 721-729. [CrossRef]

22. Yang, L.L. Multicarrier Communications; Wiley: Hoboken, NJ, USA, 2009.

23. Proakis, J.G. Digital Communication; McGrawHill: New York, NY, USA, 2001.

24. Wicker, S.B. Error Control Systems for Digital Communication and Storage; Prentice Hall: Upper Saddle River, NJ, USA, 1995.

25. Lin, S.; Costello, D.J. Error Control Coding; Pearson Prentice Hall: Upper Saddle River, NJ, USA, 2004.

26. Poisel, R.A. Electronic Warfare Receivers and Receiving Systems; Artech House: Boston, MA, USA, 2014.

(C) 2020 by the author. Licensee MDPI, Basel, Switzerland. This article is an open access article distributed under the terms and conditions of the Creative Commons Attribution (CC BY) license (http://creativecommons.org/licenses/by/4.0/). 